ARTICLE

Received 1 Oct 2012 | Accepted 14 Mar 2013 | Published 16 Apr $2013 \quad$ DOl: 10.1038/ncomms2737

\title{
Spin wave-assisted reduction in switching field of highly coercive iron-platinum magnets
}

Takeshi Seki ${ }^{1}$, Kazutoshi Utsumiya ${ }^{1}$, Yukio Nozaki ${ }^{2}$, Hiroshi Imamura ${ }^{3}$ \& Koki Takanashi ${ }^{1}$

Recent rapid progress in spintronic and magnetic storage nanodevices has required nanomagnets to balance competing goals for high coercive field and low switching field. However, a decisive route for highly efficient magnetization switching has not been established yet. Here we propose a novel switching method using a spin wave of magnetic structures twisted in a nanometre scale. We have experimentally demonstrated extremely low field-magnetization switching in a highly coercive FePt by using a spin wave excited in a soft magnetic permalloy $\left(\mathrm{Ni}_{81} \mathrm{Fe}_{19}\right)$, where permalloy is exchange-coupled to FePt through the interface. We can tune the switching field by varying the magnitude and frequency of the radio frequency magnetic field, and a significant decrease in switching field by one order of magnitude is achieved under the optimum conditions. The spin wave-assisted magnetization switching is a promising technique for ultralow-energy magnetization manipulation.

\footnotetext{
${ }^{1}$ Institute for Materials Research, Tohoku University, Sendai 980-8577, Japan. ${ }^{2}$ Department of Physics, Keio University, Yokohama 223-8522, Japan. ${ }^{3}$ National Institute of Advanced Industrial Science and Technology, Tsukuba 305-8568, Japan. Correspondence and requests for materials should be addressed to T.S. (email: go-sai@imr.tohoku.ac.jp).
} 
A route simultaneously achieving competing goals for good thermal stability of magnetization (high coercive field $H_{c}$ ) and low switching field $H_{\mathrm{sw}}$ is indispensable for the further development of spintronic and magnetic storage nanodevices $^{1}$. Energy-assisted switching ${ }^{2-12}$, spin-transfer switching ${ }^{13,14}$ and voltage-induced $H_{c}$ modulation ${ }^{15}$ are examined to overcome this predicament. One of the energy-assisted switching methods is microwave-assisted magnetization reversal (MAMR), which is considered to be a promising recording method for future ultrahigh density magnetic storage ${ }^{2-9}$. It applies a radio-frequency (rf) magnetic field $H_{\mathrm{rf}}$ to a highly coercive magnet to obtain induction of uniform magnetization precession to reduce $H_{\mathrm{sw}}$. However, MAMR has an inherent undesirable characteristic. The frequency of $H_{\mathrm{rf}}$ is around the ferromagnetic resonance (FMR) frequency $f_{\mathrm{FMR}}$, which is of the order of $100 \mathrm{GHz}$ for a highly coercive magnet. This is a serious obstacle for practical applications. Indeed, although several papers ${ }^{3-5}$ reported $H_{\text {sw }}$ reduction at single-digit gigahertz frequencies using soft magnetic materials such as a Ni-Fe alloy with $f_{\mathrm{FMR}}<10 \mathrm{GHz}$, a high excitation frequency $>10 \mathrm{GHz}$ was required in the case of a highly coercive magnet with $f_{\mathrm{FMR}}>10 \mathrm{GHz}^{7,8}$. There are no reports of clear $H_{\mathrm{SW}}$ reduction for a highly coercive magnet at single-digit gigahertz frequencies. Thus, another way of reducing $H_{\mathrm{sw}}$ while maintaining high $H_{\mathrm{c}}$ is desired. The concept of magnetization switching presented here is based on the utilization of spin wave modes in a soft magnet to switch the magnetization in a hard magnet. A similar method has been theoretically proposed by Li et al. ${ }^{16}$ and Fal et al. ${ }^{17}$ However, there has been no experimental demonstration to date as far as we are aware. Here we show the significant reduction in $H_{\mathrm{sw}}$ of highly coercive FePt due to the spin wave excitation.

\section{Results}

Magnetic structure and FMR. We combined a hard magnetic FePt layer with a soft magnetic permalloy $\left(\mathrm{Fe}_{19} \mathrm{Ni}_{81}\right.$; Py) layer, as schematically illustrated in Fig. 1a. In this FePt/Py bilayer, FePt has an uniaxial easy magnetization axis along the in-plane [001] direction. The magnetic moments in Py are spatially twisted after the magnetic field $H$ has been applied in the opposite direction to the magnetic moments in $\mathrm{FePt}^{\mathrm{I}}$. The Py layer thickness $t_{\mathrm{Py}}$ was varied in the range from 40 to $120 \mathrm{~nm}$, whereas the FePt layer thickness was fixed at $10 \mathrm{~nm}$. The full magnetization loop of an FePt/Py bilayer with $t_{\mathrm{Py}}=100 \mathrm{~nm}$ (Fig. 1b) shows two steep decreases in magnetization. The first steep decrease at around $H=-100$ Oe corresponds to the twist of magnetic moments in Py. The second steep decrease at around $H=-1,600$ Oe means that the bilayer's magnetization has switched. The minor loop is superimposed on the full loop. After all the magnetizations has
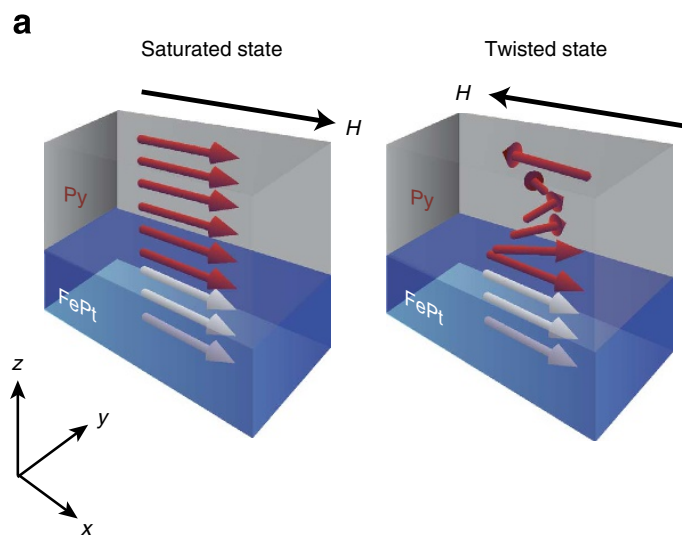

b

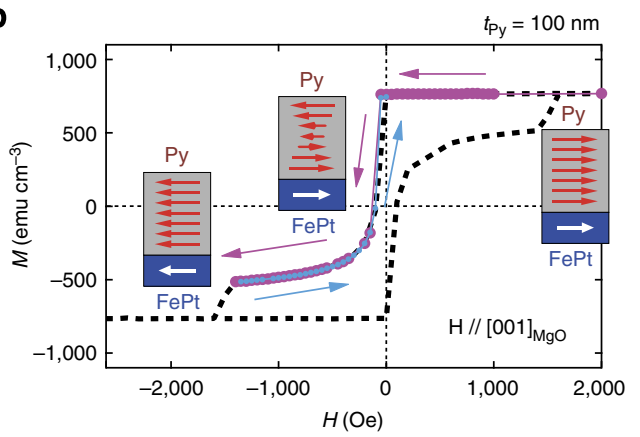

C
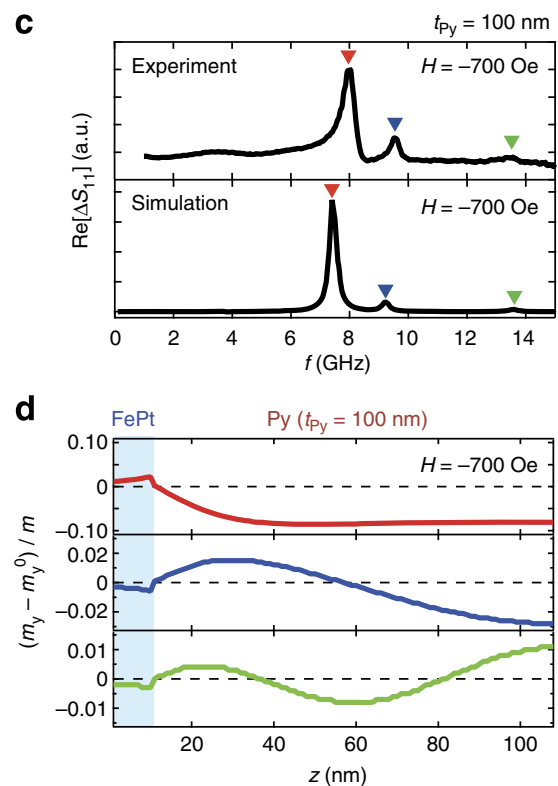

e

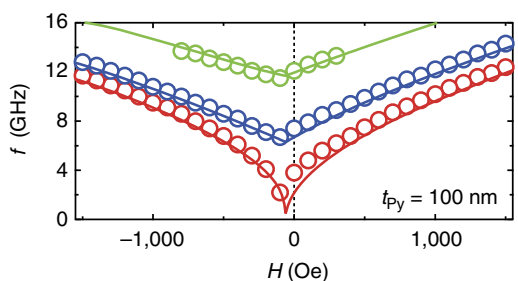

Figure 1 | Magnetization loops and FMR for an FePt/Py bilayer. (a) Illustrations of magnetic structures in an FePt/Py bilayer. The left (right) panel depicts the direction of magnetic moments in the saturated (twisted) state together with the coordinate axes. Magnetic field $H$ was applied along the in-plane [001] direction of the MgO (110) substrate, which corresponds to the easy magnetization direction of FePt. (b) Magnetization full loop (dashed line) and minor loop for the bilayer with the Py layer thickness $t_{\text {Py }}$ of $100 \mathrm{~nm}$. For the minor loop measurements, $H=50 \mathrm{kOe}$ was first applied to saturate all the magnetizations in the positive direction. Then, $H$ was swept to $-1,400$ Oe (magenta circles) and back to zero (cyan circles). (c) FMR spectra of $t_{\mathrm{Py}}=100 \mathrm{~nm}$ for experiment (upper panel) and simulation (lower panel) at $H=-700 \mathrm{Oe}$. The triangles indicate the resonant frequencies. (d) Snapshots of the deviation of magnetic moments from the equilibrium position in the $y$ direction $m_{\mathrm{y}}-m_{\mathrm{y}}^{0}$. The red, blue and green curves correspond to the first, second and third FMR peaks, respectively. These excitation modes are the PSSW modes with $n=0$ (red), $n=1$ (blue) and $n=2$ (green), where $n$ is the number of nodes. The interface between FePt and Py is located at $z=10 \mathrm{~nm}$. (e) Experimentally obtained resonant frequencies of $n=0$ (red), $n=1$ (blue) and $n=2$ (green) plotted as a function of $H$ for $t_{P y}=100 \mathrm{~nm}$ (open circles). The simulated resonant frequencies are also shown by solid lines. 
been saturated in the positive direction at $H=50 \mathrm{kOe}, H$ was swept to $-1,400 \mathrm{Oe}$ (magenta circles). Then, $H$ was returned to zero (cyan circles). The minor loop is completely reversible without any hysteresis. This behaviour is well known as springback $^{19}$, indicating that FePt and Py are exchange-coupled through the interface and that the magnetic moments in Py are spatially twisted by applying $H$.

We measured the FMR spectra of the FePt/Py bilayers using a coplanar waveguide (CPW) and a vector network analyser ${ }^{20,21}$ (see Methods). Figure 1c shows the FMR spectrum for $t_{\mathrm{Py}}=100 \mathrm{~nm}$ at $H=-700 \mathrm{Oe}$ under the application of $H_{\mathrm{rf}}=6 \mathrm{Oe}$. We observed three resonant peaks at the frequencies $f=8.0,9.5$ and $13.5 \mathrm{GHz}$, as indicated by red, blue and green triangles, respectively. The shape of the experimental FMR spectrum is well reproduced by the simulation (for more details of experimental FMR spectra, see Supplementary Fig. S1). To classify the excited magnetization dynamics at each resonance, we plotted snapshots of the deviation of magnetic moments from the equilibrium position in Fig. 1d. The red, blue and green curves correspond to the first, second and third FMR peaks, respectively. These excitation modes are known as the perpendicular standing spin wave (PSSW) modes ${ }^{22}$ with $n$ nodes. Figure 1e shows the experimental results for the $H$ dependence of resonant frequencies (open circles) together with the simulated results (solid lines). The simulated results were obtained using the calculation method reported by Livesey et al. ${ }^{23}$ (for more details, see Methods). We successfully observed the higher-order PSSW modes, which have never been reported for previous experimental studies $^{24-26}$, and the frequencies of all modes agree well with the simulation. The present results are also in good agreement with the simulation reported by Fal et al. ${ }^{17}$ Looking at Fig. 1d carefully, one can see that the dynamics of magnetic moments in FePt are also induced by PSSW. In other words, depinning occurs in FePt due to the excitation of PSSW in Py. This leads us to the idea that high $H_{\mathrm{rf}}$ enhances the amplitude of PSSW and should reduce $H_{\mathrm{sw}}$ of the FePt/Py bilayer.

Spin wave-assisted magnetization switching. We microfabricated rectangular FePt/Py bilayer elements on the signal line of a CPW (Fig. 2a) to study the $H_{\text {sw }}$ reduction by PSSW. The device resistance $R$ depends on the direction of magnetic moments in the FePt/Py bilayer owing to the anisotropic magnetoresistance effect ${ }^{27,28}$. Figure $2 \mathrm{~b}$ shows the $R-H$ curve for $t_{\mathrm{Py}}=100 \mathrm{~nm}$ without $H_{\mathrm{rf}}$ being applied. When $H$ was swept from positive to negative (denoted by red circles), $R$ showed an abrupt decrease at $H=-175 \mathrm{Oe}$, reflecting the twist of magnetic moments in Py. $R$ returned to the baseline resistance $R_{\mathrm{B}}$ at $H=H_{\mathrm{sw}}=-2,050$ Oe (green arrows), where the magnetic moments of the bilayer were switched. Figure $2 \mathrm{c}$ displays $\Delta R-H$ curves for $t_{\mathrm{Py}}=100 \mathrm{~nm}$ with $H_{\mathrm{rf}}=145 \mathrm{Oe}$, where $\Delta R$ is defined as $R-R_{\mathrm{B}}$. No remarkable changes in $H_{\mathrm{sw}}$ were observed for $f=6.0 \mathrm{GHz}$. However, the application of $H_{\mathrm{rf}}$ with $f=8.0 \mathrm{GHz}$ reduced $H_{\text {sw }}$ to $325 \mathrm{Oe}$. As $f$ was increased from 8.0 to $12.5 \mathrm{GHz}$, $H_{\text {sw }}$ monotonically increased. Small dips in $\Delta R$ appeared in the saturated state, which are indicated by orange triangles. A twodimensional map of normalized $\Delta R$ as functions of $H$ and $f$ is shown in Fig. 2d. $H$ was swept from positive to negative. The $H_{\mathrm{sw}}$ reduction was obtained in the wide range of $f$ from 8 to $14 \mathrm{GHz}$. The small dip in $\Delta R$ has the same dispersion as that of PSSW in
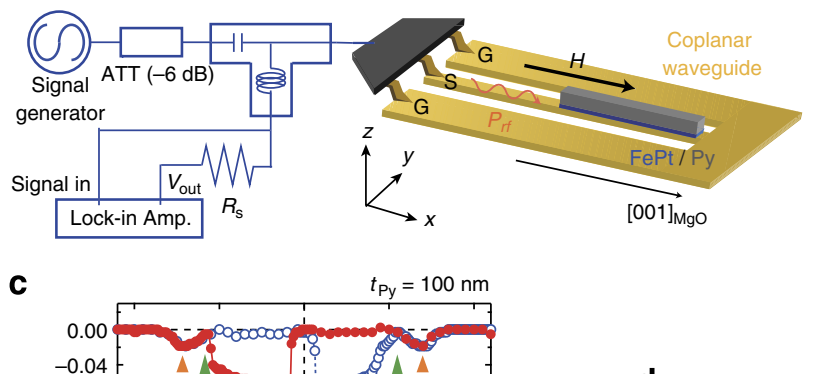

b

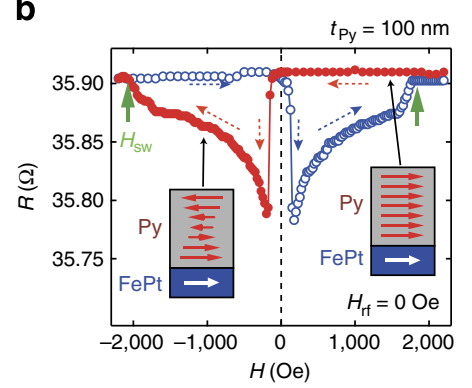

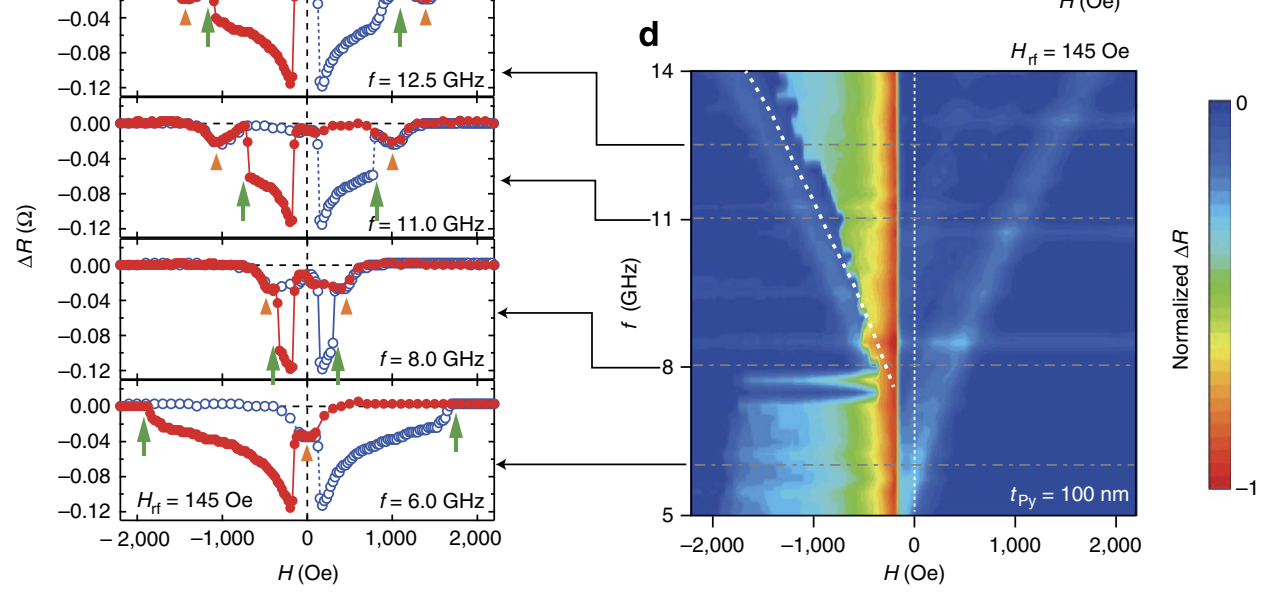

Figure 2 | Spin wave-assisted magnetization switching. (a) Schematic illustration of the device structure and measurement setup. The rf power was applied from a signal generator through the rf port of a bias-Tee. The device resistance $R$ as a function of $H$ was measured using a lock-in amplifier. $R_{\mathrm{s}}$ is a reference resistance of $1 \mathrm{k} \Omega$. The FePt/Py was designed to be rectangular, $2 \times 50 \mu \mathrm{m}^{2}$. (b) $R-H$ curve for $t_{\mathrm{Py}}=100 \mathrm{~nm}$ without the rf magnetic field $H_{\mathrm{rf}}$. The green arrows denote the switching fields $H_{\mathrm{sw}}$. (c) $\Delta R-H$ curves for $t_{\mathrm{Py}}=100 \mathrm{~nm}$ under the application of $H_{\mathrm{rf}}=145$ Oe, where $\Delta R$ is the resistance change from the baseline resistance. The frequencies $f$ of $H_{\mathrm{rf}}$ were $12.5,11.0,8.0$ and $6.0 \mathrm{GHz}$. The small dips in $\Delta R$ due to the excitation of PSSW are indicated by orange triangles. (d) Two-dimensional map of normalized $\Delta R$ as functions of $H$ and $f$ for $t_{P y}=100 \mathrm{~nm}$. $H$ was swept from positive to negative. The white dotted line denotes the resonant frequency of PSSW with $n=0$ in the twisted state of the microfabricated sample. 
the saturated state. To understand what determined the value of $H_{\text {sw }}$ at each $f$, we overlapped the resonant frequency of PSSW with $n=0$ in the twisted state of the microfabricated sample (white dotted line). Note that the frequency dependence of $H_{\mathrm{sw}}$ qualitatively followed that of the resonant magnetic field of PSSW $H_{\text {PSSW }}$, indicating that magnetization switching was induced by PSSW as we expected.

Figure 3a shows the $f$ dependence of $H_{\mathrm{sw}}$ for $t_{\mathrm{Py}}=100 \mathrm{~nm}$ obtained experimentally (circles) and numerically (squares). The experimental results qualitatively coincide with the simulation. The largest reduction in $H_{\mathrm{sw}}$ was achieved at $f=10 \mathrm{GHz}$ in the experiment, whereas the simulation showed the largest $H_{\mathrm{sw}}$ reduction at around $f=9 \mathrm{GHz}$. The simulated result has two local minima of $H_{\mathrm{sw}}$. Analysing the PSSW mode contributing to the magnetization switching, we found that these two local minima originated from the PSSW modes with different $n$. Because the experiments were performed at room temperature, these two local minima of $H_{\text {sw }}$ were smeared out. For both experiment and simulation, $H_{\mathrm{rf}}$ with low $f$ is ineffective for $H_{\mathrm{sw}}$ reduction because the resonant magnetic field at such low $f$ is so low that it cannot twist the magnetic moments enough to reduce $H_{\mathrm{sw}}$ at $H_{\mathrm{rf}}=91 \mathrm{Oe}$. Spin wave-assisted magnetization switching was also attempted for the FePt/Py bilayer with $t_{\mathrm{Py}}=40 \mathrm{~nm}$. We did not observe any $H_{\mathrm{sw}}$ reduction even when $H_{\mathrm{rf}}=113 \mathrm{Oe}$ was applied (see Supplementary Fig. S2). This result provides two important facts: the excitation of the FMR mode in FePt does not contribute to the $H_{\mathrm{sw}}$ reduction (see Supplementary Fig. S3), and a thin Py layer is not effective for $H_{\mathrm{sw}}$ reduction.

Figure $3 \mathrm{~b}$ shows $H_{\mathrm{sw}}$ as functions of $f$ and $H_{\mathrm{rf}}$ for the FePt/Py bilayer with $t_{\mathrm{Py}}=120 \mathrm{~nm}$. The frequency giving the lowest $H_{\mathrm{sw}}$ decreased as $H_{\mathrm{rf}}$ was increased. We successfully achieved the largest reduction in $H_{\text {sw }}$ from 2,000 to $250 \mathrm{Oe}$ at $f=7 \mathrm{GHz}$ and $H_{\mathrm{rf}}=134 \mathrm{Oe}$ (Fig. 3c). Let us introduce the rate of $H_{\mathrm{sw}}$ reduction defined as $\delta H_{\mathrm{sw}}=H_{\mathrm{sw}} / H_{\mathrm{sw}}^{0}$, where $H_{\mathrm{sw}}^{0}$ is $H_{\mathrm{sw}}$ without $H_{\mathrm{rf}}$. According to the MAMR calculation reported by Zhu et al. ${ }^{6}$, $H_{\mathrm{sw}}^{0}=5.3 H_{\mathrm{rf}}$ and $H_{\mathrm{sw}}=1.5 H_{\mathrm{rf}}$. Consequently, $\delta H_{\mathrm{sw}}$ for MAMR is about 0.27 , which is twice as high as that of spin wave-assisted magnetization switching, $\delta H_{\mathrm{sw}}=0.13$.

\section{Discussion}

We examined the nonlinear magnetization dynamics in the FePt/ Py bilayer because large $H_{\mathrm{rf}}$ was applied for spin wave-assisted switching. FMR spectra for the microfabricated device were also measured under the application of large $H_{\mathrm{rf}}$ (see Supplementary Fig. S4), and clear resonant peaks were observed as in the case of small $H_{\mathrm{rf}}$. The linewidth of resonant peaks became broadened and the asymmetric peak shape was observed for large $H_{\mathrm{rf}}$, which may be related to the nonlinear magnetization dynamics. However, more pronounced nonlinear effects, such as remarkable nonlinear frequency shift, were not observed. This is different from nonlinear magnetization dynamics observed in spin torque oscillators $^{29}$, where spatially uniform magnetization precession is induced by spin-polarized electric current. The precession frequency of a spin torque oscillator depends on the precession angle because the change in the precession angle leads to the large change in the effective field. On the other hand, the PSSW mode observed in this study is spatially non-uniform magnetization dynamics, and its precession frequency is mainly determined by the exchange field. The exchange field does not depend on the precession angle. Thus, the absence of the remarkable nonlinear frequency shift for the present devices is attributable to the less dependence of effective field on the precession angle.

An important point is that the PSSW modes were well defined even under the large $H_{\mathrm{rf}}$ application. To understand the magnetization dynamics during switching, the time evolution of magnetization switching behaviour was simulated numerically. Figure 4 shows the snapshots of simulated magnetic structures during magnetization switching under the application of $H_{\mathrm{rf}}=90$ Oe with $f=10 \mathrm{GHz}$ (for more details, see Supplementary Movie 1 and Supplementary Table 1), where $H$ was swept from 0 to $-1,500 \mathrm{Oe}$, and the magnetization switching occurs in the $H$
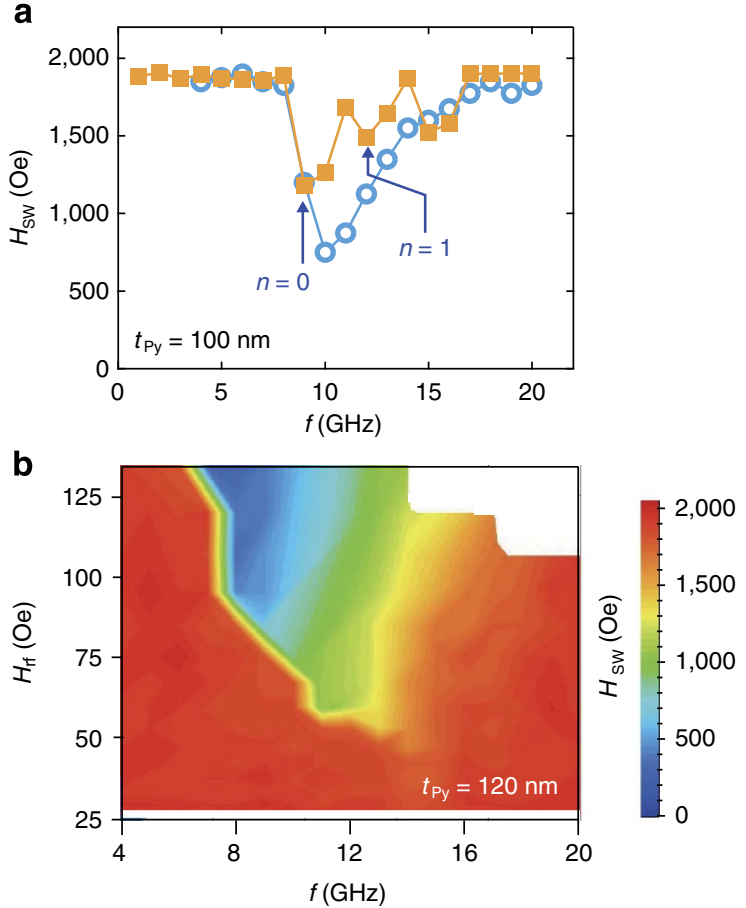

C

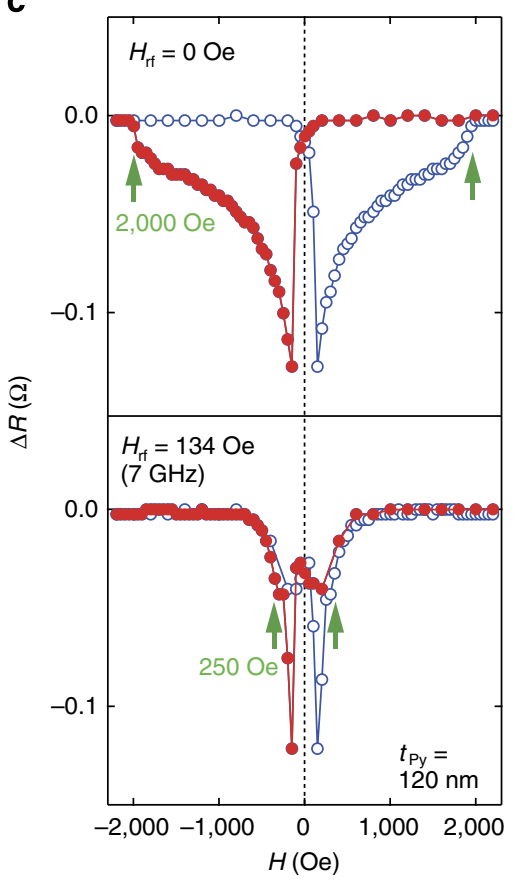

Figure 3 | Optimum conditions of $\boldsymbol{H}_{\mathbf{r f}}$ and $\boldsymbol{f}$ for $\boldsymbol{H}_{\mathbf{s w}}$ reduction. (a) $H_{\mathrm{sw}}$ as a function of $f$ for $t_{\mathrm{Py}}=100 \mathrm{~nm}$ (circles), where the value of $H_{\mathrm{rf}}$ was set to 91 Oe. Simulated $H_{\mathrm{sw}}$ as a function of $f$ is also shown by closed squares. (b) Two-dimensional map of $H_{\mathrm{sw}}$ as functions of $H_{\mathrm{rf}}$ and $f$ for $t_{\mathrm{Py}}=120 \mathrm{~nm}$. (c) $\Delta R-H$ curves for $t_{P y}=120 \mathrm{~nm}$ with $H_{\mathrm{rf}}=0 \mathrm{Oe}$ (top panel) and $134 \mathrm{Oe}$ (bottom panel). The frequency of $H_{\mathrm{rf}}$ was $7 \mathrm{GHz}$. 
range from $-1,250.5$ (Fig. 4a) to $-1,260$ Oe (Fig. 4f). The amplitude of the PSSW mode in Py is resonantly enhanced, which increases the precessional amplitude of $\mathrm{FePt}$ at the interface, and all the magnetic moments are finally switched. The PSSW mode with $n=0$ largely contributes to the $H_{\mathrm{sw}}$ reduction compared with the other modes ( $n=1$ and 2). According to the simulated result (Fig. 3a), $n=1$ could also reduce $H_{\mathrm{sw}}$ at the resonant frequency, although the $H_{\mathrm{sw}}$ reduction is not so significant and is smeared out in the experiment. A reason for the large $H_{\mathrm{sw}}$ reduction by $n=0$ is the larger precession amplitude for $n=0$ than those for $n=1$ and 2 (see Fig. 1d and Supplementary Fig. S5). As shown in Fig. 4, the large amplitude magnetization precession in Py induces the large amplitude magnetization precession in $\mathrm{FePt}$, which effectively switches the magnetization of FePt.

The numerical simulation indicates that the spin wave excitation leads to the magnetization switching in FePt. However, one might think that in the experiment, $H_{\mathrm{sw}}$ is not the real

\section{a}

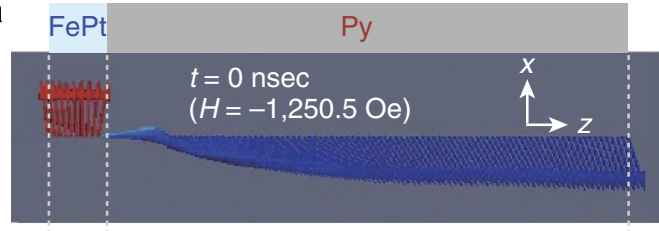

b

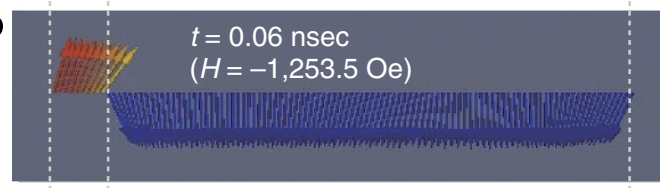

C

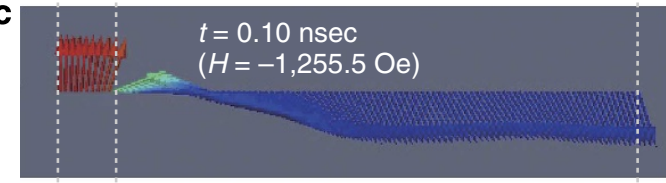

d

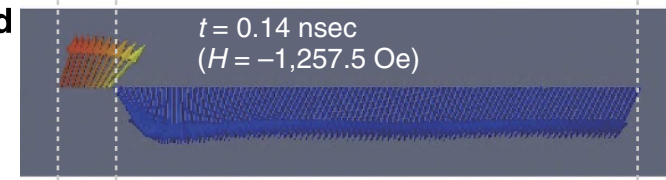

e
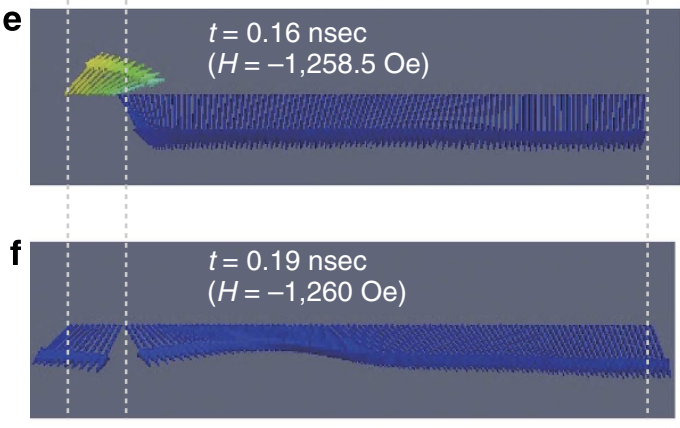

Figure 4 | Time evolution of magnetization switching behaviour.

Simulated magnetic structures in the $x-z$ plane (left panels) and the $x-y$ plane (right panels), where $H_{\mathrm{rf}}=90$ Oe with $f=10 \mathrm{GHz}$ was applied. $H$ was swept from 0 to $-1,500 \mathrm{Oe}$, and the magnetization switching occurs in the range from $H=-1,250.5 \mathrm{Oe}$ (a) to $H=-1,260 \mathrm{Oe}(\mathbf{f})$. (a-f) The timeevolved snapshots of magnetic structures during the magnetization switching. switching field of FePt, but the depinning field in FePt layer because depinning occurs in FePt under the large $H_{\mathrm{rf}}$ application. We confirmed that $H_{\mathrm{sw}}$ corresponded to the real switching field of FePt from the anisotropic magnetoresistance curves measured using different sequences of magnetic field sweep (see Supplementary Fig. S6). One might also suspect that the origin of $H_{\mathrm{sw}}$ reduction is the temperature rise due to the application of $H_{\mathrm{rf}}$, that is, Joule heating by alternating current. However, this idea can be eliminated because $R_{\mathrm{B}}$, which is a function of the device temperature, did not show any dependence on $f$ (see Supplementary Fig. S7). The large $H_{\mathrm{sw}}$ reduction by spin wave excitation was also confirmed for the other devices (see Supplementary Table S2), indicating that the error in the rate of $H_{\mathrm{sw}}$ reduction is small. Because our results simultaneously achieved the competing goals of both high $H_{\mathrm{c}}$ and low $H_{\mathrm{sw}}$, spin wave-assisted magnetization switching provides a new nanotechnology for information writing in a wide range of spintronic and magnetic storage applications.

\section{Methods}

Thin film preparation. Thin films were prepared on a $\mathrm{MgO}$ (110) single-crystal substrate. The stacking of the thin films was $\mathrm{MgO}(110)$ substrate/Fe $(1 \mathrm{~nm}) / \mathrm{Au}$ $(40 \mathrm{~nm}) / \mathrm{FePt}(10 \mathrm{~nm}) / \mathrm{Py}\left(t_{\mathrm{Py}}\right) / \mathrm{Au}(3 \mathrm{~nm})$. The layers from Fe to FePt were deposited by using an ultrahigh vacuum (UHV) magnetron sputtering system (ULVAC, Inc.) equipped with high-purity targets of Fe $(99.99 \%), \mathrm{Pt}(99.99 \%)$ and $\mathrm{Au}(99.9 \%)$. The base pressure of the UHV magnetron sputtering system was below $2 \times 10^{-7} \mathrm{~Pa}$. A 1 -nm thick Fe seed layer and a 40-nm thick Au buffer layer were deposited at ambient temperature. Subsequently, a $10-\mathrm{nm}$ thick FePt layer was deposited on the Au buffer layer after the substrate temperature was set at $450{ }^{\circ} \mathrm{C}$. Then, the film was transferred from the UHV magnetron sputtering chamber to an ion beam sputtering (IBS) system. In the IBS chamber, a Py layer followed by a 3-nm thick Au capping layer was deposited on the FePt layer at ambient temperature. The compositions of $\mathrm{FePt}$ and $\mathrm{Py}$ were determined to be $\mathrm{Fe}_{48} \mathrm{Pt}_{52}$ and $\mathrm{Ni}_{81} \mathrm{Fe}_{19}$, respectively, by electron probe $\mathrm{X}$-ray microanalysis. The epitaxial growth of $\mathrm{Au}, \mathrm{FePt}$ and Py layers with the (110) preferential crystallographic orientation was confirmed by X-ray diffraction. The X-ray diffraction patterns also indicated the formation of the $L 1_{0}$ ordered structure in the FePt layer. The magnetization loops of the thin films were measured at $295 \mathrm{~K}$ using a superconducting quantum interference device magnetometer.

FMR measurement. The FMR measurement was carried out at room temperature using a CPW and a vector network analyser. A thin film with a FePt/Py bilayer was put onto the CPW consisting of $\mathrm{Cr} / \mathrm{Au}$ layers, and the $\mathrm{CPW}$ was connected to the rf circuit employing a two-terminal $\mathrm{rf}$ probe. The $\mathrm{rf}$ power $P_{\mathrm{rf}}$ of $0 \mathrm{dBm}$ was applied to the signal line, which induced a transverse rf magnetic field of $H_{\mathrm{rf}} \sim 6 \mathrm{Oe}$, and the change in the reflected signal was detected with the vector network analyser. The value of $S_{11}$ is defined as the ratio of the reflected voltage to the input voltage. The frequency dependence of $S_{11}$ was measured under the application of $H$, which was applied along the in-plane easy magnetization direction.

Device fabrication and electrical measurement. Rectangular-shaped FePt/Py elements were prepared by using a conventional microfabrication process. First, a negative-type electron beam (EB) resist was spin-coated onto the thin film and patterned into the shape of the CPW by EB lithography (ELS-7500; Elionix Inc.). The width and length of the signal line of CPW were 4 and $50 \mu \mathrm{m}$, respectively. After exposure of the EB resist followed by development, the thin film was milled by argon ions through the resist mask. The EB resist was then spin-coated onto the CPW-shaped film and patterned into a rectangular shape with the size of $2 \times 50 \mu \mathrm{m}^{2}$, which was located on top of the signal line of the CPW-shaped film. The CPW-shaped film was again milled by argon ions. The ion milling was stopped at the top of the Au buffer layer, which was monitored by secondary ion mass spectroscopy. Finally, the Au buffer layer remained as the signal and ground lines of the CPW, and the rectangular FePt/Py element was prepared on the signal line. This rectangular shape induces the shape anisotropy of Py. Because the long axis of rectangle is the same as the in-plane [001] direction of the FePt layer, both uniaxial easy magnetization axes of FePt and $\mathrm{Py}$ are in the same direction. The microfabricated device was connected to the rf-compatible circuit via a two-terminal rf probe, and $P_{\mathrm{rf}}$ was applied from a signal generator through the rf port of a bias-Tee after $-6 \mathrm{~dB}$ attenuation. The application of $P_{\mathrm{rf}}$ induced $H_{\mathrm{rf}}$ in the $y-z$ plane of the $\mathrm{FePt} / \mathrm{Py}$ element. The device resistance was simultaneously measured using a lockin amplifier.

Numerical simulation. Numerical simulation was carried out by solving the Landau-Lifshitz-Gilbert (LLG) equation by using the conventional fourth-order 
Runge-Kutta algorithm. The effective magnetic field in the LLG equation is the vector sum of the anisotropy field, magnetostatic field, exchange field, $H$, and $H_{\mathrm{rf}}$. In the present numerical study, we considered an exchange-coupled $\mathrm{FePt} / \mathrm{Py}$ bilayer of infinite size in the film plane. The thicknesses of FePt and Py were fixed at 10 and $98 \mathrm{~nm}$, respectively. The film was discretized into $1-\mathrm{nm}$ thick infinite plates, and each plate was assumed to have a uniform magnetization vector. The saturation magnetizations of FePt and Py were determined to be 1000 and $800 \mathrm{emu} \mathrm{cm}^{-3}$, respectively, from the magnetization measurement. The inter- and intra-layer exchange constants of FePt/Py, $A_{\text {int }}, A_{\mathrm{FePt}}$ and $A_{\mathrm{Py}}$, were chosen to be $1.0 \times 10^{-7}, 1.0 \times 10^{-6}$ and $1.3 \times 10^{-6} \mathrm{erg} \mathrm{cm}^{-1}$, respectively, so as to reproduce the experimental magnetization loop. The small value of $A_{\text {int }}$ results from the slight oxidation and/or the contamination on the FePt surface during the sample transfer from the UHV magnetron sputtering system to the IBS system with breaking the vacuum. The uniaxial anisotropy constant of FePt $K_{\mathrm{FePt}}$ was assumed to be $3.3 \times 10^{6} \mathrm{erg} \mathrm{cm}^{-3}$, which was determined from the magnetic field required to switch FePt in the lithographically patterned device (see Fig. $2 \mathrm{~b}$ ), whereas $K_{\mathrm{Py}}$ was set to $1.0 \times 10^{3} \mathrm{erg} \mathrm{cm}^{-3}$. One may consider that $K_{\mathrm{FePt}}=3.3 \times 10^{6} \mathrm{erg} \mathrm{cm}^{-3}$ is small. The one-dimensional model often leads to the underestimation of $K_{\mathrm{FePt}}$ to reproduce $H_{\mathrm{sw}}$ because in experiment $H_{\mathrm{sw}}$ is generally much smaller than the anisotropy field. The experimental value of $K_{\mathrm{FePt}}$ was about $2.5 \times 10^{7} \mathrm{erg} \mathrm{cm}^{-3}$ The Gilbert damping constants for Py and FePt were 0.008 and 0.02 , respectively. To evaluate the $H_{\mathrm{sw}}$ reduction under the $H_{\mathrm{rf}}$ application, we set $H_{\mathrm{rf}}$ to 90 Oe. When $H_{\mathrm{sw}}$ as a function of $f$ was evaluated, the magnetization was initially magnetized along $+x$ direction. The magnetization was relaxed for $10 \mathrm{~ns}$ under $H_{\mathrm{rf}}$ applied along the $y$ axis. The magnetization curve under the $H_{\mathrm{rf}}$ application was then calculated sweeping the dc magnetic field along the $x$ direction. The field sweep velocity was $50 \mathrm{Oens}^{-1}$. $H_{\mathrm{sw}}$ was defined as the magnetic field at which the magnetization of the FePt layer became zero. To obtain the $f$ dependence of $H_{\mathrm{sw}}$, $f$ was varied in the range from 1 to $20 \mathrm{GHz}$.

Using the same parameters as those for the magnetization switching simulation, we calculated the stable magnetic structure at each $H$ by solving the LLG equation. For these stable magnetic structures, we solved the eigenvalue equations corresponding to the linear response to the small $H_{\mathrm{rf}}$ and obtained the resonant frequencies. To construct the eigenvalue equations, we employed the local rotated frame where the coordinates are rotated about the $z$ axis such that the new $x$ axis points in the same direction as the local magnetization vectors.

\section{References}

1. Chappert, C., Fert, A. \& Nguyen Van Dau, F. The emergency of spin electronics in data storage. Nat. Mater. 6, 813-823 (2007).

2. Thirion, C., Wernsdorfer, W. \& Mailly, D. Switching of magnetization by nonlinear resonance studied in single nanoparticles. Nat. Mater. 2, 524-527 (2003).

3. Woltersdorf, G. \& Back, C. H. Microwave assisted switching of single domain $\mathrm{Ni}_{80} \mathrm{Fe}_{20}$ elements. Phys. Rev. Lett. 99, 227207 (2007).

4. Nembach, H. T. et al. Microwave assisted switching in a $\mathrm{Ni}_{81} \mathrm{Fe}_{19}$ ellipsoid. Appl. Phys. Lett. 90, 062503 (2007).

5. Moriyama, T. et al. Microwave-assisted magnetization switching of $\mathrm{Ni80Fe} 20$ in magnetic tunnel junctions. Appl. Phys. Lett. 90, 152503 (2007).

6. Zhu, J. -G., Zhu, X. \& Tang, Y. Microwave assisted magnetic recording. IEEE Trans. Magn. 44, 125-131 (2008)

7. Nozaki, Y., Narita, N., Tanaka, T. \& Matsuyama, K. Microwave-assisted magnetization reversal in a $\mathrm{Co} / \mathrm{Pd}$ multilayer with perpendicular magnetic anisotropy. Appl. Phys. Lett. 95, 082505 (2009).

8. Yoshioka, T. et al. Microwave-assisted magnetization reversal in a perpendicularly magnetized film. Appl. Phys. Exp. 3, 013002 (2010).

9. Boone, C. T., Katine, J. A., Marinero, E. E., Pisana, S. \& Terris, B. D. Microwave-assisted magnetic reversal in perpendicular media. IEEE Magn. Lett. 3, 3500104 (2012).

10. Atkinson, D. \& Cowburn, R. P. Heat-assisted magnetization switching in elongated submicrometer Permalloy structures. Appl. Phys. Lett. 85, 1386-1388 (2009).

11. Rottmayer, R. E. et al. Heat-assisted magnetic recording. IEEE Trans. Magn. 42, 2417-2421 (2006).

12. Prejbeanu, I. L. et al. Thermally assisted MRAM. J. Phys.: Condens. Matter 19, 165218 (2007).

13. Tsoi, M. et al. Excitation of a magnetic multilayer by an electric current. Phys. Rev. Lett. 80, 4281-4284 (1998).
14. Myers, E. B., Ralph, D. C., Katine, J. A., Louie, R. N. \& Buhrman, R. A. Currentinduced switching of domains in magnetic multilayer devices. Science 285, 867-870 (1999).

15. Weisheit, M. et al. Electric field-induced modification of magnetism in thinfilm ferromagnets. Science 315, 349-351 (2007).

16. Li, S. et al. Microwave assisted magnetization reversal in composite media. Appl. Phys. Lett. 94, 202509 (2009).

17. Fal, T. J., Livesey, K. L. \& Camley, R. E. Domain wall and microwave assisted switching in an exchange spring bilayer. J. Appl. Phys. 109, 093911 (2011).

18. Utsumiya, K., Seki, T. \& Takanashi, K. Magnetic properties of $L 1_{0}-\mathrm{FePt} /$ permalloy exchange-spring films. J. Appl. Phys. 110, 103911 (2011)

19. Kneller, E. F. \& Hawig, R. The exchange-spring magnet: a new material principle for permanent magnets. IEEE Trans. Magn. 27, 3588-3600 (1991).

20. Counil, G. et al. Spin wave contributions to the high-frequency magnetic response of thin films obtained with inductive methods. J. Appl. Phys. 95, 56465652 (2004).

21. Novosad, V. et al. Magnetic vortex resonance in patterned ferromagnetic dots. Phys. Rev. B 72, 024455 (2005)

22. Demokritov, S. O., Hillebrands, B. \& Slavin, A. N. Brillouin light scattering studies of confined spin waves: linear and nonlinear confinement. Phys. Reports 348, 441-489 (2001).

23. Livesey, K. L., Crew, D. C. \& Stamps, R. L. Spin wave valve in an exchange spring bilayer. Phys. Rev. B 73, 184432 (2006).

24. Fullerton, E. E., Jiang, J. S., Grimsditch, M., Sowers, C. H. \& Bader, S. D. Exchange-spring behavior in epitaxial hard/soft magnetic bilayers. Phys. Rev. B 58, 12193-12200 (1998).

25. Grimsditch, M. et al. Exchange-spring systems: coupling of hard and soft ferromagnets as measured by magnetization and Brillouin light scattering. J. Appl. Phys. 85, 5901-5904 (1999).

26. Schmool, D. S., Apolinário, A., Casoli, F. \& Albertini, F. Ferromagnetic resonance study of $\mathrm{Fe} / \mathrm{FePt}$ coupled films with perpendicular anisotropy. IEEE Trans. Magn. 44, 3087-3090 (2008).

27. Takanashi, K., Kamiguchi, Y., Fujimori, H. \& Motokawa, M. Magnetization and magnetoresistance of Fe/ Gd ferrimagnetic multilayer films. J. Phys. Soc. Jpn. 61, 3721-3731 (1992).

28. Mibu, K., Nagahama, T., Shinjo, T. \& Ono, T. Magnetoresistance of Bloch-walltype magnetic structures induced in $\mathrm{NiFe} / \mathrm{CoSm}$ exchange-spring bilayers. Phys. Rev. B 58, 6442-6446 (1998).

29. Kiselev, S. I. et al. Microwave oscillations of a nanomagnet driven by a spinpolarized current. Nature 425, 380-383 (2003).

\section{Acknowledgements}

This work was partially supported by a Grant-in-Aid for Young Scientists B (23760659) and Research and Development for Next-Generation Information Technology from MEXT. The device fabrication was partly performed at Advanced Research Center of Metallic Glasses, IMR, Tohoku University. We thank H. Muraoka for helpful comments and Y. Murakami for technical assistance.

\section{Author contributions}

T.S. and K.T. planned and supervised the study. T.S. and K.U. carried out the sample preparation and the measurement of magnetic and transport properties. Y.N. and H.I. contributed to the theoretical modelling for the interpretation and performed the micromagnetic simulation. All of the authors contributed to the physical understanding, data analysis and manuscript preparation.

\section{Additional information}

Supplementary Information accompanies this paper at http://www.nature.com/ naturecommunications

Competing financial interests: The authors declare no competing financial interests.

Reprints and permission information is available online at http://npg.nature.com/ reprintsandpermissions/

How to cite this article: Seki, T. et al. Spin wave-assisted reduction in switching field of highly coercive FePt magnets. Nat. Commun. 4:1726 doi: 10.1038/ncomms2737 (2013). 